\title{
Cobalt(III)-Catalyzed Diastereoselective Three-Component C-H Bond Addition to Butadiene and Activated Ketones
}

\author{
Zican Shen \\ Chen Li \\ Brandon Q. Mercado \\ Jonathan A. Ellman* (D)
}

Department of Chemistry, Yale University, New Haven, CT 06520, USA

jonathan.ellman@yale.edu

Published as part of the Special Topic

Domino C-H Functionalization Reaction/Cascade Catalysis

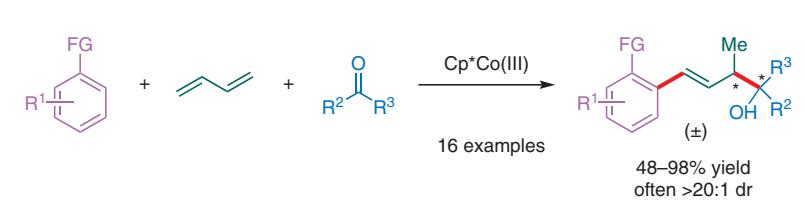

Received: 02.10.2019

Accepted after revision: 22.10.2019

Published online: 07.11.2019

DOI: 10.1055/s-0039-1690741; Art ID: ss-2019-c0566-st

Abstract A highly diastereoselective three-component $\mathrm{C}-\mathrm{H}$ bond addition across butadiene and activated ketones is described. This transformation provides homoallylic tertiary alcohols through the formation of two $\mathrm{C}-\mathrm{C} \sigma$-bonds and with complete selectivity for an $\mathrm{E}$-alkene isomer. The reaction exhibits good scope with respect to activated ketone inputs, including highly strained cyclic and electron-deficient cyclic and acyclic ketones. Additionally, high diastereoselectivities were achieved for alcohols prepared from unsymmetrical ketones.

Key words $\mathrm{C}-\mathrm{H}$ activation, cobalt, ketones, diastereoselectivity, multicomponent reaction, homogeneous catalysis

Transition-metal-catalyzed $\mathrm{C}-\mathrm{H}$ bond additions to a large variety of coupling partners provide a robust approach to assemble synthetically useful motifs. ${ }^{1}$ While reaction of a $\mathrm{C}-\mathrm{H}$ bond substrate with two different coupling partners potentially represents a powerful approach to access complex structures, relatively few examples have been reported to date. The Catellani reaction initiated by Pd-catalyzed oxidative addition of an aryl halide has proven to be an effective strategy to accomplish three-component additions. ${ }^{2}$ We and others have recently explored an alternative approach that proceeds by $\mathrm{C}-\mathrm{H}$ activation followed by sequential coupling with two different coupling partners. ${ }^{3}$ Relevant to the study described in this article, previous work by our lab ${ }^{3 e, h}$ and others ${ }^{3 f}$ has demonstrated that $\mathrm{Co}(\mathrm{III})-$ and $\mathrm{Rh}(\mathrm{III})$-catalyzed three-component $\mathrm{C}-\mathrm{H}$ bond additions with butadiene, monosubstituted or 1,2-disubstituted dienes, and aldehydes provide homoallylic alcohols with high diastereoselectivity (Scheme 1, eq. 1, 2).

Ketones are inherently more stable and sterically congested than aldehydes, and consequently, direct $\mathrm{C}-\mathrm{H}$ bond additions even to activated ketones have only rarely been reported. ${ }^{4}$ Recently, our lab reported the first examples of

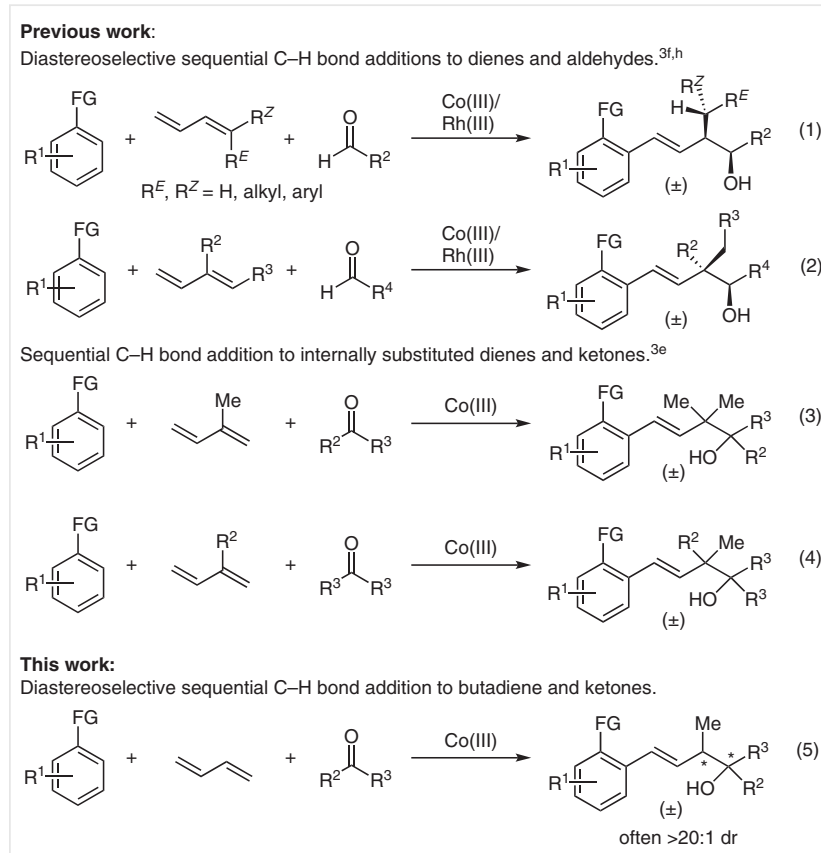

Scheme 1 Transition-metal-catalyzed three-component addition to dienes and carbonyls

Co(III)-catalyzed three-component coupling to internally substituted dienes and activated ketones (eq. 3, 4). However, only those diene and ketone combinations that gave products with a single stereogenic center were investigated. To expand upon these first examples of intermolecular sequential $\mathrm{C}-\mathrm{H}$ bond additions to dienes and ketones, we sought to evaluate a broader array of ketones, in particular for diastereoselective additions to give products with two stereogenic centers. Herein, we describe a $\mathrm{Co}(\mathrm{III})$-catalyzed three-component $\mathrm{C}-\mathrm{H}$ bond addition to butadiene and activated ketones to provide homoallylic tertiary alcohols with 
the formation of two new C-C $\sigma$-bonds (eq. 5). Notably, for the first time, alcohols bearing two stereogenic centers can be obtained with high diastereoselectivity for three-component additions with both cyclic and acyclic unsymmetrical ketones.

We began by exploring the reaction parameters for the coupling of benzamide 1a with butadiene (2) and ethyl benzoylformate (3a), which proved to be one of the more challenging ketone coupling partners (vide infra). Under the optimized reaction conditions that had previously been adopted for the coupling of butadiene and aldehydes (Table 1 , entry 1$),{ }^{3 \mathrm{~h}}$ none of the desired three-component product was observed, and only $13 \%$ of a product resulting from two-component $\mathrm{C}-\mathrm{H}$ bond addition to butadiene was obtained. Considering the greater stability and steric encumbrance of ketones relative to aldehydes, a higher reaction temperature was next investigated. At $70{ }^{\circ} \mathrm{C}, 20 \%$ of threecomponent product $\mathbf{4 a}$ was obtained as a single diastereomer (entry 2). However, at a higher reaction temperature of $90{ }^{\circ} \mathrm{C}$, the yield of $\mathbf{4 a}$ dropped to $12 \%$ (entry 3). Substrate concentration was next evaluated, with $1.0 \mathrm{M}$ leading to lower conversion than $0.4 \mathrm{M}$ (entry 4 ), while $0.2 \mathrm{M}$ provided a modest increase in the yield of $\mathbf{4 a}$ to $25 \%$ (entry 5). Finally, additives that have been employed in other $\mathrm{Co}$ (III)catalyzed $\mathrm{C}-\mathrm{H}$ addition reactions were examined as well. ${ }^{3 \mathrm{k}, 5}$ Although replacing $\mathrm{AcOH}$ with LiOAc led to no conversion (entry 6), PivOH was a superior additive for this transformation and provided $\mathbf{4 a}$ in $50 \%$ yield (entry 7 ).

Having optimized the reaction conditions, we next explored the scope for the ketone coupling partner with benzamide $\mathbf{1 a}$ as the $\mathrm{C}-\mathrm{H}$ bond substrate (Scheme 2). In addition to ethyl benzoylformate (3a), highly strained fourmembered cyclic ketones also provided homoallylic tertiary alcohols in good to excellent yields. $N$-Carboxybenzyl (Cbz)- protected 3-azetidinone and 3-oxetanone furnished products $\mathbf{4 b}$ and $\mathbf{4 c}$ in $92 \%$ and $83 \%$ yield, respectively. Additionally, reaction with less activated cyclobutanone gave alcohol 4d in 50\% yield. Electron-deficient isatin provided homoallylic alcohol $\mathbf{4 e}$ with two contiguous stereogenic centers in high yield and with high diastereoselectivity. Additions with $\mathrm{N}$-methylisatin and $\mathrm{N}$-benzylisatin afforded products $\mathbf{4 f}$ and $\mathbf{4 g}$ in $66 \%$ and $82 \%$ yield, respectively, also with high diastereoselectivity. Moreover, more electron-deficient 6-chloroisatin derivatives afforded alcohols $\mathbf{4 h}$ and $4 \mathbf{i}$ in near quantitative yields and with $>20: 1 \mathrm{dr}$.

Next, additional $\mathrm{C}-\mathrm{H}$ bond substrates 1 were investigated for several different ketones $\mathbf{3}$ (Scheme 3 ). In addition to tertiary benzamide 1a, the secondary $N$-methylbenzamide is also an effective $\mathrm{C}-\mathrm{H}$ bond substrate, coupling with 1$\mathrm{Cbz}-3$-azetidinone to give product $\mathbf{4 j}$ in $86 \%$ yield. The secondary $N$-methylbenzamide also reacted with the acyclic ketone ethyl benzoylformate to furnish product $\mathbf{4 k}$ in $74 \%$ yield and with $>20: 1 \mathrm{dr}$. An X-ray crystal structure of the major diastereomer of alcohol $4 \mathbf{k}$ rigorously established the relative configuration of the methyl and hydroxyl groups for this ketone coupling partner. $N$-Phenylpyrazole with a methyl substituent at the meta position coupled with $\mathrm{N}$ methylisatin to provide tertiary alcohol $\mathbf{4 1}$ in $48 \%$ yield and with high diastereoselectivity. The relative stereochemistry for $\mathbf{4 1}$ was confirmed by X-ray crystallography, thereby providing the stereochemical relationship for isatin-derived products. Pyrimidine also served as an excellent directing group as demonstrated for three-component addition with ethyl benzoylformate to afford alcohol $\mathbf{4 m}$ in $70 \%$ yield and with >20:1 dr. However, a blocking group, such as a methyl group at the meta position, is necessary to prevent bisfunctionalization. The 2-phenylpyrimidine $\mathrm{C}-\mathrm{H}$ bond substrate is also effective for coupling with $\mathrm{N}$-Cbz-3-azetidinone

Table 1 Screening of Reaction Conditions ${ }^{\mathrm{a}}$
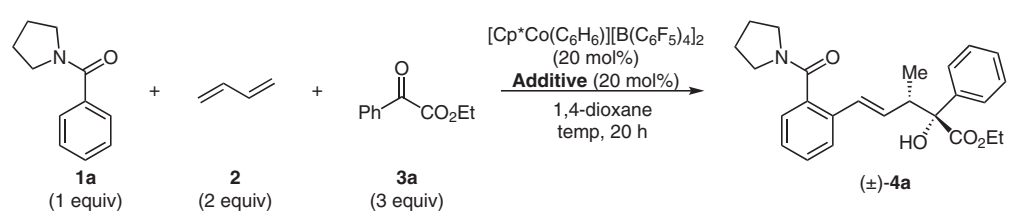

\begin{tabular}{|c|c|c|c|c|}
\hline Entry & Additive & Temp $\left({ }^{\circ} \mathrm{C}\right)$ & Concn (M) & Yield $^{b}(\%)$ of $4 a$ \\
\hline 1 & $\mathrm{AcOH}$ & 50 & 0.4 & 0 \\
\hline 2 & $\mathrm{AcOH}$ & 70 & 0.4 & 20 \\
\hline 3 & $\mathrm{AcOH}$ & 90 & 0.4 & 12 \\
\hline 4 & $\mathrm{AcOH}$ & 70 & 1.0 & 15 \\
\hline 5 & $\mathrm{AcOH}$ & 70 & 0.2 & 25 \\
\hline 6 & $\mathrm{LiOAC}$ & 70 & 0.2 & 0 \\
\hline 7 & $\mathrm{PivOH}$ & 70 & 0.2 & 50 \\
\hline
\end{tabular}

a Reaction conditions: 1a (0.1 mmol), 2 (0.2 mmol), $3 a(0.3 \mathrm{mmol})$.

${ }^{\mathrm{b}}$ Yields determined by ${ }^{1} \mathrm{H}$ NMR analysis relative to $1,3,5$-trimethoxybenzene. 

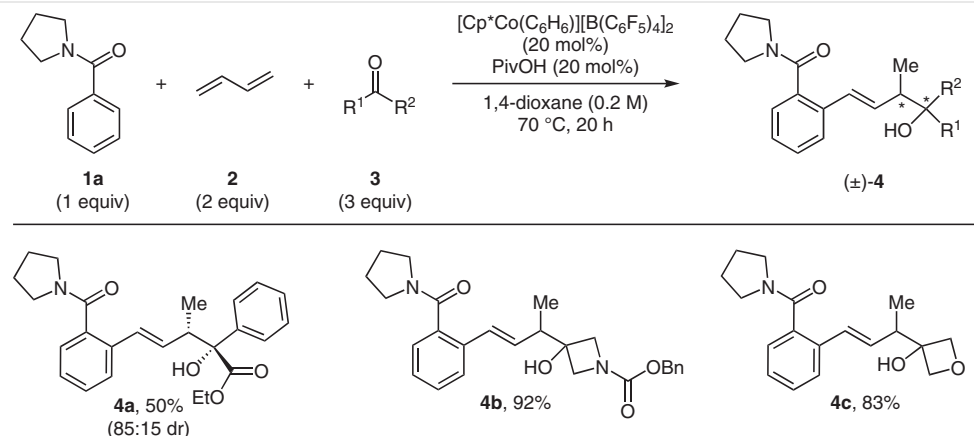<smiles>[M]C(/C=C/c1ccccc1C(=O)N1CCCC1)C1(O)CCC1</smiles><smiles>[Y4]C(/C=C/c1ccccc1C(=O)N1CCCC1)[C@]1(O)C(=O)Nc2ccccc21</smiles><smiles>CC(/C=C/c1ccccc1C(=O)N1CCCC1)[C@@]([14CH3])(O)C(=O)n1c(=O)n(C)c2ccccc21</smiles>
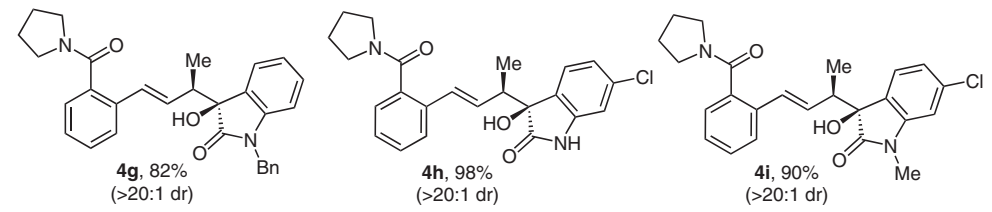

Scheme 2 Three-component coupling of benzamide 1a, butadiene (2), and diverse ketones $\mathbf{3}$. Reactions were performed at $0.1 \mathrm{mmol}$ scale; isolated yields of products after purification by chromatography.

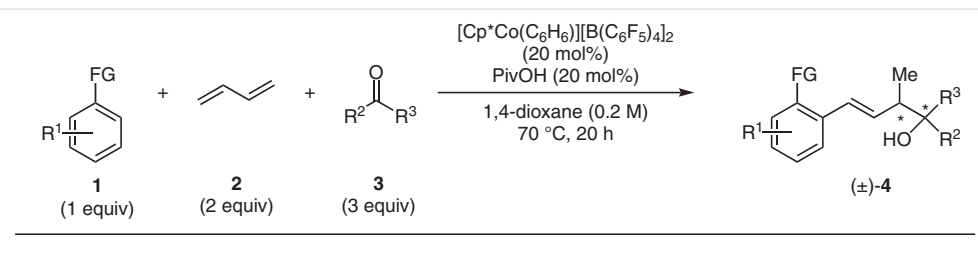<smiles>C[M]C(/C=C/c1ccccc1C(=O)O)C1(O)CN(C(=O)OCc2ccccc2)C1</smiles>
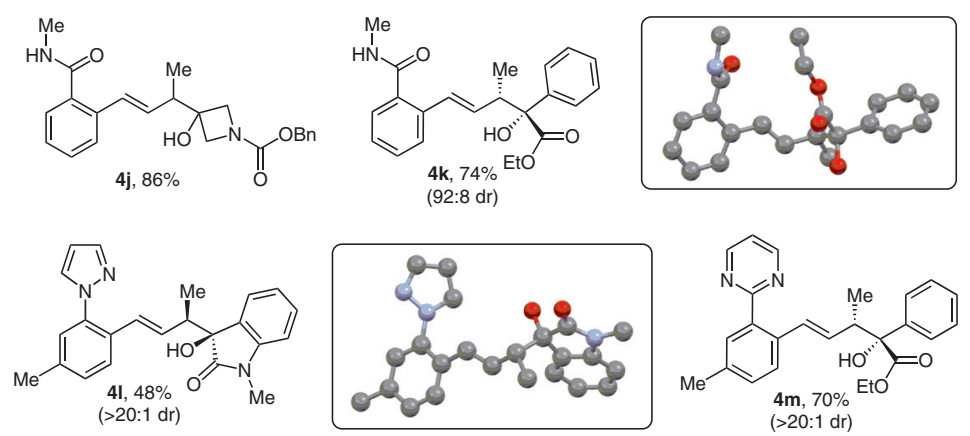<smiles>Cc1ccc(/C=C/C(C)C2(O)CN(C(=O)OCc3ccccc3)C2)c(-c2ncccn2)c1</smiles><smiles>[Y10][C@H](/C=C/c1ccc(C)cc1-c1ncccn1)[C@]1(O)C(=O)Nc2ccc([N+](=O)[O-])cc21</smiles>

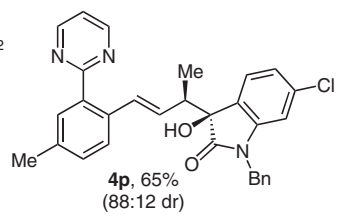

Scheme 3 Three-component coupling of $\mathrm{C}-\mathrm{H}$ bond substrates $\mathbf{1}$ with butadiene (2) and selected ketones $\mathbf{3}$. Reactions were performed at $0.1 \mathrm{mmol}$ scale; isolated yields of products after purification by chromatography. For the X-ray crystal structures of $\mathbf{4} \mathbf{k}$ and $\mathbf{4 l}$, hydrogen atoms are omitted for clarity. 
to furnish alcohol $\mathbf{4 n}$. Isatin derivatives like 5-nitroisatin and $\mathrm{N}$-benzyl-6-chloroisatin afforded the corresponding products $\mathbf{4 0}$ and $\mathbf{4 p}$ in $82 \%$ and $65 \%$ yield, respectively, with good diastereoselectivity.

To evaluate the scalability of the reaction, the threecomponent product $\mathbf{4 b}$ was prepared at a 15 -fold larger scale (Scheme 4). Reaction of benzamide $\mathbf{1 a}$ with butadiene (2) and 1-Cbz-3-azetidinone (3b) at $1.5 \mathrm{mmol}$ scale provided product $\mathbf{4 b}$ in $95 \%$ yield, which is consistent with the yield obtained for the corresponding smaller scale reaction.

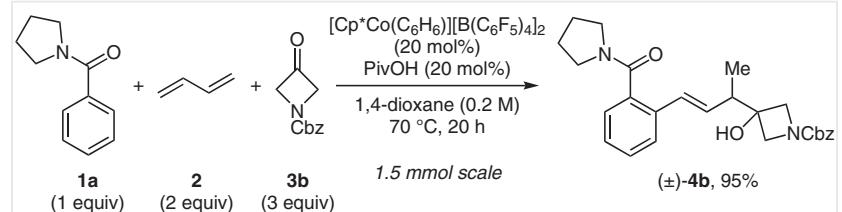

Scheme 4 Three-component coupling of benzamide 1a, butadiene (2), and 1-Cbz-3-azetidinone (3b) on $1.5 \mathrm{mmol}$ scale

A catalytic cycle is depicted in Scheme 5, which is based on prior mechanistic studies for three-component additions to dienes and aldehydes. ${ }^{3 e, h}$ The $\mathrm{Co}(\mathrm{III})$-catalyzed C-H bond activation of 1 through concerted metalation-deprotonation forms metallacycle A. Migratory insertion into butadiene subsequently affords Co-allyl species $\mathbf{B}$. To obtain the necessary connectivity observed in the product, $\beta$-hydride elimination at $\mathrm{C} 1$ then provides $\mathrm{Co}$-diene complex $\mathbf{C}$, which undergoes alkene insertion into the $\mathrm{Co}-\mathrm{H}$ bond to afford Co-allyl species $\mathbf{D}$. Diastereoselective addition of intermediate $\mathbf{D}$ with ketone $\mathbf{3}$ via a chair-like transition state depicted in E, followed by protonolysis to release the catalyst, would then provide product 4 .

In conclusion, we have developed a $\mathrm{Co}(\mathrm{III})$-catalyzed three-component $\mathrm{C}-\mathrm{H}$ bond addition to butadiene and ketones. Efficient coupling was observed for a number of different activated ketones and $\mathrm{C}-\mathrm{H}$ bond substrates. Notably, $\mathrm{Co}(\mathrm{III})$-catalyzed diastereoselective additions with unsymmetrical ketones have been demonstrated for the first time. In addition, the relative stereochemistry of the two stereogenic centers in the three-component addition products for isatins and ethyl benzoylformate was rigorously established by X-ray crystallography.

All $\mathrm{Co}$ (III)-catalyzed reactions were set up in a $\mathrm{N}_{2}$-filled glovebox, using glassware that was oven-dried $\left(150^{\circ} \mathrm{C}\right)$ and evacuated while hot prior to use. Solvents were sparged with argon and purified by elution through a column of activated alumina under argon before use and were stored with 3 Å molecular sieves in a $\mathrm{N}_{2}$-filled glovebox (molecular sieves were dried at $200{ }^{\circ} \mathrm{C}$ overnight under vacuum). Unless otherwise noted, all reagents were purchased from commercial sources and used without further purification. Microwave vials and caps were purchased from Biotage (part number 351521 and 352298, respectively). Product purification was performed by preparative TLC with plates from Analtech $\left(1 \mathrm{~mm}\right.$ silica gel, $20 \times 20 \mathrm{~cm}$ ). ${ }^{1} \mathrm{H}$ and ${ }^{13} \mathrm{C}$ NMR

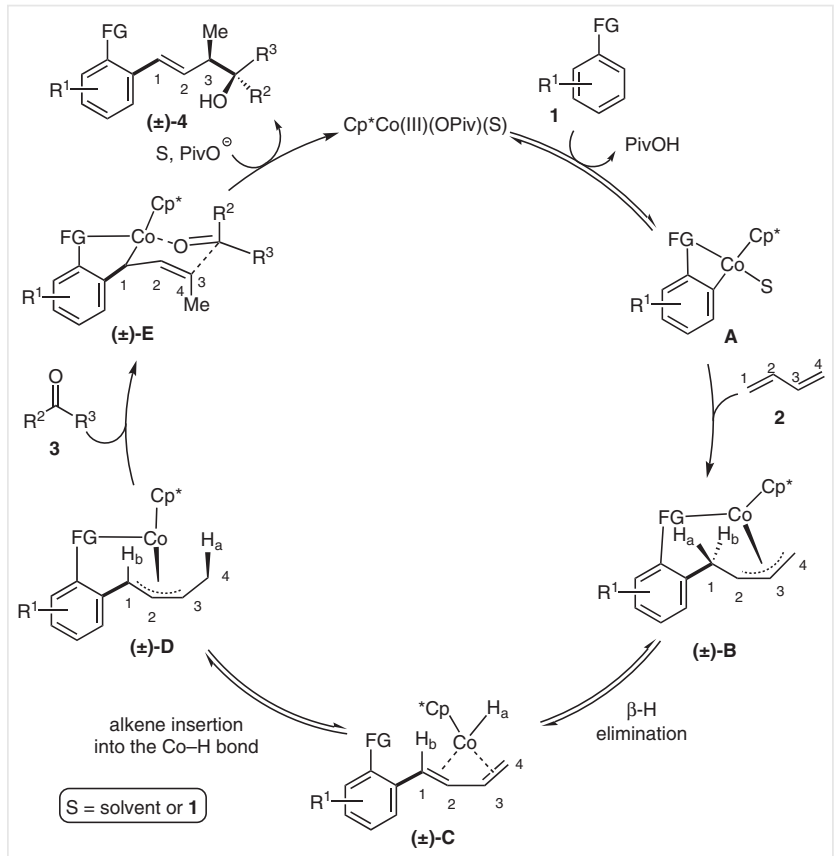

Scheme 5 Proposed mechanism for the three-component transformation

spectra were recorded on a 400,500 , or $600 \mathrm{MHz}$ instrument. Partial IR spectra are reported. High-resolution mass spectra were obtained using electrospray ionization (ESI) on a time-of-flight mass spectrometer.

Low-temperature X-ray diffraction data ( $\omega$-scans) were collected on a Rigaku MicroMax-007HF diffractometer coupled to a Saturn994+ CCD detector with $\mathrm{Cu} \mathrm{K \alpha}$ radiation $(\lambda=1.54178 \AA$ ) for the structures of $\mathbf{4 k}$ and 41 . $^{6}$ The diffraction images were processed and scaled using Rigaku Oxford Diffraction software (CrysAlis ${ }^{\mathrm{Pro}}$ ). The structures were solved with SHELXT and refined against $F^{2}$ on all data by full-matrix least squares with SHELXL. ${ }^{7}$ For the full numbering scheme of compounds $4 \mathbf{k}$ and $\mathbf{4 1}$, see the full details of the X-ray crystal structure determination (CIF) in the Supporting Information, Section III.

\section{Homoallylic Tertiary Alcohols 4; General Procedure}

In a $\mathrm{N}_{2}$-filled glovebox, a $2.0 \mathrm{~mL}$ Biotage microwave vial with a triangular stir bar $(1 \times 0.5 \mathrm{~cm})$ was charged with the indicated $\mathrm{C}-\mathrm{H}$ bond partner $\left(0.100 \mathrm{mmol}, 1.00\right.$ equiv) and $\left[\mathrm{Cp} * \mathrm{Co}\left(\mathrm{C}_{6} \mathrm{H}_{6}\right)\right]\left[\mathrm{B}\left(\mathrm{C}_{6} \mathrm{~F}_{5}\right)_{4}\right]_{2}(32.6$ $\mathrm{mg}, 0.0200 \mathrm{mmol}, 0.200$ equiv). Following this, the corresponding ketone $(0.300 \mathrm{mmol}, 3.00$ equiv) was added. A $0.2 \mathrm{M}$ solution of pivalic acid in 1,4-dioxane ( $100 \mu \mathrm{L}, 0.0200 \mathrm{mmol}, 0.200$ equiv) and 1,4-dioxane $(350 \mu \mathrm{L})$ were added successively. At last, a commercially available $4 \mathrm{M}$ stock solution of butadiene in THF $(50 \mu \mathrm{L}, 0.200 \mathrm{mmol}, 2.00$ equiv) was added. The reaction vial was then sealed and taken outside the glovebox. The reaction mixture was stirred in a preheated oil bath at $70{ }^{\circ} \mathrm{C}$ for $20 \mathrm{~h}$. The reaction vial was then cooled to room temperature and uncapped. The reaction was quenched with saturated $\mathrm{NaHCO}_{3}(10 \mathrm{~mL})$, and the resulting mixture was then extracted with EtOAc $(5 \times 3 \mathrm{~mL})$. The organic layers were combined and dried over $\mathrm{Na}_{2} \mathrm{SO}_{4}$. After filtration of the mixture through a Celite plug using EtOAc as the eluent, the resulting mixture was then concentrated and purified by the indicated chromatographic method to afford the desired product. 
Preparation of Homoallylic Tertiary Alcohol $4 \mathrm{~b}$ on $1.5 \mathrm{mmol}$ Scale In a $\mathrm{N}_{2}$-filled glovebox, a $30 \mathrm{~mL}$ Biotage microwave vial with a football-shaped stir bar $(2 \times 0.5 \mathrm{~cm})$ was charged with phenyl(pyrrolidin1-yl)methanone (1a; $263 \mathrm{mg}, 1.50 \mathrm{mmol}, 1.00$ equiv) and $\left[\mathrm{Cp}^{*} \mathrm{Co}\left(\mathrm{C}_{6} \mathrm{H}_{6}\right)\right]\left[\mathrm{B}\left(\mathrm{C}_{6} \mathrm{~F}_{5}\right)_{4}\right]_{2}$ (489 mg, $0.300 \mathrm{mmol}, 0.200$ equiv). Following this, benzyl 3-oxoazetidine-1-carboxylate $(923 \mathrm{mg}, 4.50 \mathrm{mmol}$, 3.00 equiv) was added. A $0.2 \mathrm{M}$ solution of pivalic acid in 1,4-dioxane (1500 $\mu \mathrm{L}, 0.300 \mathrm{mmol}, 0.200$ equiv) and 1,4-dioxane $(5.25 \mathrm{~mL})$ were added successively. At last, a $4 \mathrm{M}$ stock solution of butadiene in THF ( $750 \mu \mathrm{L}, 3.00 \mathrm{mmol}, 2.00$ equiv) was added. The reaction vial was then sealed and taken outside the glovebox. The reaction mixture was stirred in a preheated oil bath at $70{ }^{\circ} \mathrm{C}$ for $20 \mathrm{~h}$. The reaction vial was then cooled to room temperature and uncapped. The reaction was quenched with saturated $\mathrm{NaHCO}_{3}(50 \mathrm{~mL})$, and the resulting mixture was then extracted with EtOAc $(5 \times 15 \mathrm{~mL})$. The organic layers were combined and dried over $\mathrm{Na}_{2} \mathrm{SO}_{4}$. After filtration of the mixture through a medium fritted funnel using EtOAc as the eluent, the resulting mixture was then concentrated and purified by silica gel chromatography (50\% EtOAc in hexane) to provide $\mathbf{4 b}$ ( $619 \mathrm{mg}$, 95\% yield) as a yellow oil. Both the ${ }^{1} \mathrm{H}$ NMR and ${ }^{13} \mathrm{C}$ NMR spectra are in perfect agreement with the corresponding spectra of $\mathbf{4 b}$ obtained according to the general procedure (see the Supporting Information, Section II).

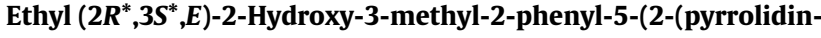
1-ylcarbonyl)phenyl)pent-4-enoate (4a)

The general procedure was followed using phenyl(pyrrolidin-1yl)methanone (1a; $17.5 \mathrm{mg}, 0.100 \mathrm{mmol}, 1.00$ equiv) and ethyl 2-oxo2-phenylacetate ( $53.4 \mathrm{mg}, 0.300 \mathrm{mmol}, 3.00$ equiv). Purification by silica gel chromatography (50\% EtOAc in hexane) provided 4 a (20.4 mg, $50 \%$ yield; $85: 15 \mathrm{dr}$ ) as a colorless oil.

IR (neat): 3491, 2975, 2876, 1724, 1615, 1449, $1425 \mathrm{~cm}^{-1}$.

${ }^{1} \mathrm{H} \mathrm{NMR}\left(500 \mathrm{MHz}, \mathrm{CDCl}_{3}\right.$ ): $\delta=7.68(\mathrm{~d}, J=7.3 \mathrm{~Hz}, 1.67 \mathrm{H}$, major), 7.62 (d, $J=7.3 \mathrm{~Hz}, 0.32 \mathrm{H}$, minor), $7.49(\mathrm{~d}, J=8.2 \mathrm{~Hz}, 1 \mathrm{H}), 7.39-7.32(\mathrm{~m}$, $2 \mathrm{H}), 7.32-7.27(\mathrm{~m}, 2 \mathrm{H}), 7.23(\mathrm{~m}, 2 \mathrm{H}), 6.59(\mathrm{~d}, J=15.8 \mathrm{~Hz}, 0.85 \mathrm{H}$, major), 6.28 (dd, $J=15.9,8.8 \mathrm{~Hz}, 0.85 \mathrm{H}$, major), 6.23 ( $\mathrm{m}, 0.15 \mathrm{H}$, minor), 6.03 (dd, $J=15.9,8.9 \mathrm{~Hz}, 0.15 \mathrm{H}$, minor), 4.35-4.08 (m, $2 \mathrm{H}), 3.83(\mathrm{~s}$, $1 \mathrm{H}), 3.68(\mathrm{t}, J=7.1 \mathrm{~Hz}, 1.74 \mathrm{H}$, major), $3.59(\mathrm{~m}, 0.36 \mathrm{H}$, minor), 3.27 (m, $1 \mathrm{H}), 3.14-3.05(\mathrm{~m}, 2 \mathrm{H}), 1.95(\mathrm{~m}, 2 \mathrm{H}), 1.87-1.81(\mathrm{~m}, 2 \mathrm{H}), 1.31(\mathrm{t}$, $J=7.2 \mathrm{~Hz}, 0.51 \mathrm{H}$, minor), $1.23(\mathrm{t}, J=7.1 \mathrm{~Hz}, 2.71 \mathrm{H}$, major), $1.15(\mathrm{~d}, J=$ $6.8 \mathrm{~Hz}, 0.44 \mathrm{H}$, minor), 0.88 (d, $J=6.8 \mathrm{~Hz}, 2.56 \mathrm{H}$, major).

${ }^{13} \mathrm{C}$ NMR $\left(126 \mathrm{MHz}, \mathrm{CDCl}_{3}\right): \delta=174.9,169.5,140.2,136.5,133.7$, 133.3, 132.5, 129.0, 128.8, 128.4, 128.14, 128.06, 127.9, 127.6, 127.44, 127.40, 127.1, 126.2, 126.1, 126.0, 125.9, 125.6, 80.5, 62.7, 60.4, 48.3, 47.8, 45.7, 45.5, 45.3, 26.0, 25.8, 24.6, 24.5, 15.9, 14.4, 14.13, 14.07; peaks for both major and minor isomer are listed.

HRMS (ESI): $m / z[\mathrm{M}+\mathrm{H}]^{+}$calcd for $\mathrm{C}_{25} \mathrm{H}_{30} \mathrm{NO}_{4}{ }^{+}$: 408.2169; found: 408.2175 .

\section{Benzyl (E)-3-Hydroxy-3-(4-(2-(pyrrolidin-1-ylcarbonyl)- phenyl)but-3-en-2-yl)azetidine-1-carboxylate (4b)}

The general procedure was followed using $\mathbf{1 a}(17.5 \mathrm{mg}, 0.100 \mathrm{mmol}$, 1.00 equiv) and benzyl 3-oxoazetidine-1-carboxylate ( $61.5 \mathrm{mg}, 0.300$ mmol, 3.00 equiv). Purification by silica gel chromatography ( $50 \%$ EtOAc in hexane) provided $\mathbf{4 b}$ ( $40.0 \mathrm{mg}, 92 \%$ yield) as a yellow oil.

IR (neat): 3390, 2964, 2879, 2362, 1700, 1607, 1462, 1429, $1356 \mathrm{~cm}^{-1}$. ${ }^{1} \mathrm{H} \mathrm{NMR}\left(400 \mathrm{MHz}, \mathrm{CDCl}_{3}\right): \delta=7.40(\mathrm{~d}, J=7.7 \mathrm{~Hz}, 1 \mathrm{H}), 7.36-7.27(\mathrm{~m}$, $5 \mathrm{H}), 7.24(\mathrm{~m}, 1 \mathrm{H}), 7.23-7.15(\mathrm{~m}, 2 \mathrm{H}), 6.48(\mathrm{~d}, J=15.9 \mathrm{~Hz}, 1 \mathrm{H}), 6.06$ (dd, $J=15.9,8.2 \mathrm{~Hz}, 1 \mathrm{H}), 5.04(\mathrm{~s}, 2 \mathrm{H}), 3.92(\mathrm{~m}, 2 \mathrm{H}), 3.78(\mathrm{~m}, 2 \mathrm{H})$, $3.59(\mathrm{~m}, 2 \mathrm{H}), 3.06(\mathrm{~m}, 2 \mathrm{H}), 2.60-2.47(\mathrm{~m}, 1 \mathrm{H}), 1.98-1.71(\mathrm{~m}, 5 \mathrm{H})$, $1.07(\mathrm{~d}, J=6.8 \mathrm{~Hz}, 3 \mathrm{H})$.
${ }^{13} \mathrm{C}$ NMR $\left(101 \mathrm{MHz}, \mathrm{CDCl}_{3}\right): \delta=169.9,156.6,136.4,135.7,133.7$, 132.7, 129.3, 128.9, 128.5, 128.1, 127.9, 127.5, 126.5, 126.1, 72.6, 66.9, $60.8,60.5,48.6,45.7,44.4,25.9,24.5,13.5$.

HRMS (ESI): $m / z[\mathrm{M}+\mathrm{H}]^{+}$calcd for $\mathrm{C}_{26} \mathrm{H}_{31} \mathrm{~N}_{2} \mathrm{O}_{4}{ }^{+}$: 435.2278; found: 435.2284 .

\section{(E)-(2-(3-(3-Hydroxyoxetan-3-yl)but-1-en-1-yl)phenyl)(pyrroli-} din-1-yl)methanone (4c)

The general procedure was followed using $1 \mathbf{a}(17.5 \mathrm{mg}, 0.100 \mathrm{mmol}$, 1.00 equiv) and oxetan-3-one ( $21.6 \mathrm{mg}, 0.300 \mathrm{mmol}, 3.00$ equiv). $\mathrm{Pu}-$ rification by silica gel chromatography (50\% EtOAc in hexane) provided 4 c (24.9 $\mathrm{mg}, 83 \%$ yield $)$ as a colorless oil.

IR (neat): 3375, 2962, 2874, 1606, 1452, 1430, $1340 \mathrm{~cm}^{-1}$.

${ }^{1} \mathrm{H} \mathrm{NMR}\left(500 \mathrm{MHz}, \mathrm{CDCl}_{3}\right): \delta=7.45(\mathrm{~d}, J=7.7 \mathrm{~Hz}, 1 \mathrm{H}), 7.37-7.26(\mathrm{~m}$, $1 \mathrm{H}), 7.27-7.17(\mathrm{~m}, 2 \mathrm{H}), 6.52$ (d, $J=15.9 \mathrm{~Hz}, 1 \mathrm{H}), 6.12$ (dd, $J=15.9$, $8.2 \mathrm{~Hz}, 1 \mathrm{H}), 4.51(\mathrm{~m}, 4 \mathrm{H}), 3.63(\mathrm{t}, J=7.1 \mathrm{~Hz}, 2 \mathrm{H}), 3.10(\mathrm{t}, J=6.8 \mathrm{~Hz}$, $2 \mathrm{H}), 2.77(\mathrm{~m}, 1 \mathrm{H}), 1.93(\mathrm{~m}, 2 \mathrm{H}), 1.83(\mathrm{~m}, 2 \mathrm{H}), 1.11$ (d, $J=6.8 \mathrm{~Hz}$, $3 \mathrm{H})$.

${ }^{13} \mathrm{C}$ NMR $\left(126 \mathrm{MHz}, \mathrm{CDCl}_{3}\right): \delta=169.7,136.2,133.7,132.8,129.1$, 128.8, 127.4, 126.4, 126.2, 82.4, 82.3, 76.1, 48.4, 45.6, 43.7, 26.0, 24.6, 13.6.

HRMS (ESI): $m / z[\mathrm{M}+\mathrm{H}]^{+}$calcd for $\mathrm{C}_{18} \mathrm{H}_{24} \mathrm{NO}_{3}{ }^{+}$: 302.1751; found: 302.1756.

(E)-(2-(3-(1-Hydroxycyclobutyl)but-1-en-1-yl)phenyl)(pyrrolidin1-yl)methanone (4d)

The general procedure was followed using 1a $(17.5 \mathrm{mg}, 0.100 \mathrm{mmol}$, 1.00 equiv) and cyclobutanone ( $21.0 \mathrm{mg}, 0.300 \mathrm{mmol}, 3.00$ equiv). Purification by silica gel chromatography (50\% EtOAc in hexane) provided $4 d$ ( $15.0 \mathrm{mg}, 50 \%$ yield) as a colorless oil.

IR (neat): 3396, 2971, 2876, 1611, 1452, $1428 \mathrm{~cm}^{-1}$.

${ }^{1} \mathrm{H}$ NMR $\left(500 \mathrm{MHz}, \mathrm{CDCl}_{3}\right): \delta=7.48(\mathrm{~d}, J=7.8 \mathrm{~Hz}, 1 \mathrm{H}), 7.30(\mathrm{~m}, 1 \mathrm{H})$, $7.23(\mathrm{~m}, 2 \mathrm{H}), 6.50(\mathrm{~d}, J=15.9 \mathrm{~Hz}, 1 \mathrm{H}), 6.20(\mathrm{dd}, J=15.9,8.2 \mathrm{~Hz}, 1 \mathrm{H})$, $3.65(\mathrm{t}, J=7.0 \mathrm{~Hz}, 2 \mathrm{H}), 3.10(\mathrm{t}, J=6.8 \mathrm{~Hz}, 2 \mathrm{H}), 2.53-2.44(\mathrm{~m}, 1 \mathrm{H})$, 2.20-2.12 (m, 2 H), 1.98-1.89 (m, 4 H), 1.83 (m, 4 H), 1.09 (d, J = 6.9 $\mathrm{Hz}, 3 \mathrm{H})$.

${ }^{13} \mathrm{C}$ NMR $\left(126 \mathrm{MHz}, \mathrm{CDCl}_{3}\right): \delta=169.8,136.3,134.2,134.0,129.0$, 128.1, 127.2, 126.2, 126.1, 77.4, 48.3, 45.5, 44.9, 34.3, 34.0, 25.9, 24.6, $13.5,12.1$.

HRMS (ESI): $m / z[\mathrm{M}+\mathrm{H}]^{+}$calcd for $\mathrm{C}_{19} \mathrm{H}_{26} \mathrm{NO}_{2}{ }^{+}$: 300.1958; found: 300.1964 .

(E)-3-Hydroxy-3-(4-(2-(pyrrolidin-1-ylcarbonyl)phenyl)but-3-en2-yl)indolin-2-one (4e)

The general procedure was followed using 1a $(17.5 \mathrm{mg}, 0.100 \mathrm{mmol}$, 1.00 equiv) and indoline-2,3-dione (44.1 $\mathrm{mg}, 0.300 \mathrm{mmol}, 3.00$ equiv). Purification by silica gel chromatography (70\% EtOAc in hexane) provided 4 e $(21.0 \mathrm{mg}, 55 \%$ yield; $>20: 1 \mathrm{dr})$ as a yellow oil.

IR (neat): 3271, 2974, 2324, 1712, 1594, 1462, $1433 \mathrm{~cm}^{-1}$.

${ }^{1} \mathrm{H}$ NMR (400 MHz, $\mathrm{CDCl}_{3}$ ): $\delta=9.28(\mathrm{~s}, 1 \mathrm{H}), 7.48(\mathrm{~d}, J=7.7 \mathrm{~Hz}, 1 \mathrm{H})$, $7.31(\mathrm{t}, J=7.5 \mathrm{~Hz}, 1 \mathrm{H}), 7.27-7.24(\mathrm{~m}, 1 \mathrm{H}), 7.23-7.19(\mathrm{~m}, 1 \mathrm{H}), 7.13$ $(\mathrm{m}, 2 \mathrm{H}), 6.92(\mathrm{t}, J=7.5 \mathrm{~Hz}, 1 \mathrm{H}), 6.70(\mathrm{~d}, J=7.7 \mathrm{~Hz}, 1 \mathrm{H}), 6.60(\mathrm{~d}, J=$ $15.8 \mathrm{~Hz}, 1 \mathrm{H}), 6.27(\mathrm{dd}, J=15.8,8.1 \mathrm{~Hz}, 1 \mathrm{H}), 4.99(\mathrm{~s}, 1 \mathrm{H}), 3.58(\mathrm{~m}$, $2 \mathrm{H}), 3.16(\mathrm{~m}, 1 \mathrm{H}), 3.11-3.01(\mathrm{~m}, 1 \mathrm{H}), 2.91(\mathrm{~m}, 1 \mathrm{H}), 1.79(\mathrm{~m}, 4 \mathrm{H})$, $0.75(\mathrm{~d}, J=6.7 \mathrm{~Hz}, 3 \mathrm{H})$.

${ }^{13} \mathrm{C}$ NMR $\left(101 \mathrm{MHz}, \mathrm{CDCl}_{3}\right): \delta=180.9,170.0,141.2,136.1,133.8$, $131.5,129.8,129.5,129.2,128.6,127.5,126.0,125.1,122.7,110.5$, 79.4, 48.5, 45.7, 44.0, 25.8, 24.5, 14.0 . 
HRMS (ESI): $m / z[M+\mathrm{H}]^{+}$calcd for $\mathrm{C}_{23} \mathrm{H}_{25} \mathrm{~N}_{2} \mathrm{O}_{3}{ }^{+}$: 377.1860; found: 377.1865 .

\section{(E)-3-Hydroxy-1-methyl-3-(4-(2-(pyrrolidin-1-ylcarbonyl)- phenyl)but-3-en-2-yl)indolin-2-one (4f)}

The general procedure was followed using $1 \mathrm{a}(17.5 \mathrm{mg}, 0.100 \mathrm{mmol}$, 1.00 equiv) and 1-methylindoline-2,3-dione $(48.3 \mathrm{mg}, 0.300 \mathrm{mmol}$, 3.00 equiv). Purification by silica gel chromatography (50\% EtOAc in hexane) provided $\mathbf{4 f}(25.7 \mathrm{mg}, 66 \%$ yield; $>20: 1 \mathrm{dr})$ as a yellow powder.

IR (neat): 3351, 2972, 2361, 1706, 1612, 1512, $1461 \mathrm{~cm}^{-1}$.

${ }^{1} \mathrm{H}$ NMR $\left(400 \mathrm{MHz}, \mathrm{CDCl}_{3}\right): \delta=7.46(\mathrm{~d}, J=7.8 \mathrm{~Hz}, 1 \mathrm{H}), 7.30(\mathrm{~m}, 2 \mathrm{H})$, 7.23-7.10 (m, $2 \mathrm{H}), 7.04(\mathrm{~d}, J=7.6 \mathrm{~Hz}, 1 \mathrm{H}), 6.99(\mathrm{t}, J=7.6 \mathrm{~Hz}, 1 \mathrm{H})$, $6.77(\mathrm{~d}, J=7.8 \mathrm{~Hz}, 1 \mathrm{H}), 6.47(\mathrm{~d}, J=15.6 \mathrm{~Hz}, 1 \mathrm{H}), 6.19(\mathrm{dd}, J=15.7,8.8$ Hz, $1 \mathrm{H}), 3.38(\mathrm{~m}, 2 \mathrm{H}), 3.17(\mathrm{~m}, 1 \mathrm{H}), 3.05(\mathrm{~m}, 1 \mathrm{H}), 2.99(\mathrm{~s}, 3 \mathrm{H}), 2.72$ $(\mathrm{m}, 1 \mathrm{H}), 1.79(\mathrm{~m}, 4 \mathrm{H}), 0.64(\mathrm{~d}, J=6.7 \mathrm{~Hz}, 3 \mathrm{H})$.

${ }^{13} \mathrm{C}$ NMR $\left(101 \mathrm{MHz}, \mathrm{CDCl}_{3}\right): \delta=178.5,170.0,143.1,134.8,133.8$, $131.2,130.1,129.9,129.7,127.9,127.6,126.3,126.1,124.7,123.2$, 108.7, 79.1, 49.2, 45.8, 45.0, 26.0, 25.8, 24.3, 14.2.

HRMS (ESI): $m / z[\mathrm{M}+\mathrm{H}]^{+}$calcd for $\mathrm{C}_{24} \mathrm{H}_{27} \mathrm{~N}_{2} \mathrm{O}_{3}{ }^{+}$: 391.2016; found: 391.2022 .

\section{(E)-1-Benzyl-3-hydroxy-3-(4-(2-(pyrrolidin-1-ylcarbonyl)- phenyl)but-3-en-2-yl)indolin-2-one (4g)}

The general procedure was followed using $1 \mathbf{a}(17.5 \mathrm{mg}, 0.100 \mathrm{mmol}$, 1.00 equiv) and 1-benzylindoline-2,3-dione $(71.1 \mathrm{mg}, 0.300 \mathrm{mmol}$, 3.00 equiv). Purification by silica gel chromatography (70\% EtOAc in hexane) provided $\mathbf{4 g}$ (38.3 $\mathrm{mg}, 82 \%$ yield; $>20: 1 \mathrm{dr}$ ) as a yellow powder.

IR (neat): 3353, 2974, 1715, 1610, 1465, 1453, 1430, $1359 \mathrm{~cm}^{-1}$.

${ }^{1} \mathrm{H}$ NMR $\left(500 \mathrm{MHz}, \mathrm{CDCl}_{3}\right): \delta=7.49(\mathrm{~d}, J=7.7 \mathrm{~Hz}, 1 \mathrm{H}), 7.41-7.30(\mathrm{~m}$, $2 \mathrm{H}), 7.30-7.19(\mathrm{~m}, 7 \mathrm{H}), 7.19-7.13(\mathrm{~m}, 1 \mathrm{H}), 6.98(\mathrm{~m}, 1 \mathrm{H}), 6.69(\mathrm{~m}$, $1 \mathrm{H}), 6.55(\mathrm{~d}, J=16.1 \mathrm{~Hz}, 1 \mathrm{H}), 6.38(\mathrm{dd}, J=15.9,7.4 \mathrm{~Hz}, 1 \mathrm{H}), 5.03(\mathrm{~d}$, $J=15.8 \mathrm{~Hz}, 1 \mathrm{H}), 4.69(\mathrm{~d}, J=15.8 \mathrm{~Hz}, 1 \mathrm{H}), 3.58(\mathrm{~m}, 2 \mathrm{H}), 3.05(\mathrm{~m}, 3 \mathrm{H})$, $1.85(\mathrm{~m}, 2 \mathrm{H}), 1.76(\mathrm{~m}, 2 \mathrm{H}), 0.88(\mathrm{~d}, J=6.7 \mathrm{~Hz}, 3 \mathrm{H})$.

${ }^{13} \mathrm{C}$ NMR $\left(126 \mathrm{MHz}, \mathrm{CDCl}_{3}\right): \delta=177.9,169.6,143.0,136.5,135.5$, 133.7, 131.6, 131.5, 129.7, 129.5, 129.1, 128.8, 127.6, 127.5, 127.3, $127.1,126.3,126.0,124.9,122.9,109.3,78.7,48.3,45.6,44.4,43.9$, 25.9, 24.5, 13.9 .

HRMS (ESI): $m / z[\mathrm{M}+\mathrm{H}]^{+}$calcd for $\mathrm{C}_{30} \mathrm{H}_{31} \mathrm{~N}_{2} \mathrm{O}_{3}{ }^{+}$: 467.2329; found: 467.2335 .

\section{(E)-6-Chloro-3-hydroxy-3-(4-(2-(pyrrolidin-1-ylcarbonyl)-} phenyl)but-3-en-2-yl)indolin-2-one (4h)

The general procedure was followed using $1 \mathrm{a}(17.5 \mathrm{mg}, 0.100 \mathrm{mmol}$, 1.00 equiv) and 6 -chloroindoline-2,3-dione $(54.5 \mathrm{mg}, 0.300 \mathrm{mmol}$, 3.00 equiv). Purification by silica gel chromatography (50\% EtOAc in hexane) provided $\mathbf{4 h}$ (40.3 $\mathrm{mg}$, $98 \%$ yield; $>20: 1 \mathrm{dr}$ ) as a red powder.

IR (neat): 3267, 2973, 2361, 1719, 1613, $1455 \mathrm{~cm}^{-1}$.

${ }^{1} \mathrm{H}$ NMR $\left(500 \mathrm{MHz}, \mathrm{CDCl}_{3}\right): \delta=9.70(\mathrm{~s}, 1 \mathrm{H}), 7.51(\mathrm{~d}, J=7.8 \mathrm{~Hz}, 1 \mathrm{H})$, $7.32(\mathrm{t}, J=7.5 \mathrm{~Hz}, 1 \mathrm{H}), 7.27-7.20(\mathrm{~m}, 2 \mathrm{H}), 7.12(\mathrm{~d}, J=7.9 \mathrm{~Hz}, 1 \mathrm{H})$, $6.90(\mathrm{~d}, J=7.9 \mathrm{~Hz}, 1 \mathrm{H}), 6.84(\mathrm{~s}, 1 \mathrm{H}), 6.61(\mathrm{~d}, J=15.8 \mathrm{~Hz}, 1 \mathrm{H}), 6.28(\mathrm{dd}$, $J=15.8,8.1 \mathrm{~Hz}, 1 \mathrm{H}), 5.28(\mathrm{~s}, 1 \mathrm{H}), 3.63(\mathrm{~m}, 2 \mathrm{H}), 3.15(\mathrm{~m}, 1 \mathrm{H}), 3.09-$ $3.01(\mathrm{~m}, 1 \mathrm{H}), 3.00-2.89(\mathrm{~m}, 1 \mathrm{H}), 1.82(\mathrm{~m}, 4 \mathrm{H}), 0.80(\mathrm{~d}, J=6.7 \mathrm{~Hz}$, $3 \mathrm{H})$.

${ }^{13} \mathrm{C}$ NMR $\left(126 \mathrm{MHz}, \mathrm{CDCl}_{3}\right): \delta=181.0,169.8,142.7,136.3,135.0$, 133.6, 131.2, 130.0, 129.2, 127.5, 127.4, 126.1, 126.0, 125.9, 122.5, $111.2,79.1,48.4,45.7,43.9,25.8,24.5,14.1$.
HRMS (ESI): $m / z[\mathrm{M}+\mathrm{H}]^{+}$calcd for $\mathrm{C}_{23} \mathrm{H}_{24} \mathrm{ClN}_{2} \mathrm{O}_{3}{ }^{+}: 411.1470$; found: 411.1476 .

(E)-6-Chloro-3-hydroxy-1-methyl-3-(4-(2-(pyrrolidin-1-ylcarbonyl)phenyl)but-3-en-2-yl)indolin-2-one (4i)

The general procedure was followed using $\mathbf{1 a}(17.5 \mathrm{mg}, 0.100 \mathrm{mmol}$, 1.00 equiv) and 6-chloro-1-methylindoline-2,3-dione $(58.7 \mathrm{mg}, 0.300$ mmol, 3.00 equiv). Purification by silica gel chromatography $(50 \%$ EtOAc in hexane) provided $\mathbf{4 i}$ (38.2 $\mathrm{mg}, 90 \%$ yield; >20:1 dr) as a yellow powder.

IR (neat): 3364, 2968, 2877, 2362, 2339, 1733, 1609, $1456 \mathrm{~cm}^{-1}$.

${ }^{1} \mathrm{H}$ NMR $\left(500 \mathrm{MHz}, \mathrm{CDCl}_{3}\right): \delta=7.49(\mathrm{~d}, J=7.8 \mathrm{~Hz}, 1 \mathrm{H}), 7.34(\mathrm{~m}, 1 \mathrm{H})$, 7.28-7.16 (m, $3 \mathrm{H}), 6.98(\mathrm{~d}, J=7.5 \mathrm{~Hz}, 1 \mathrm{H}), 6.80(\mathrm{~s}, 1 \mathrm{H}), 6.58(\mathrm{~d}, J=$ $15.9 \mathrm{~Hz}, 1 \mathrm{H}), 6.31$ (dd, $J=15.9,7.7 \mathrm{~Hz}, 1 \mathrm{H}), 4.20-3.92(\mathrm{~m}, 1 \mathrm{H}), 3.59$ $(\mathrm{m}, 2 \mathrm{H}), 3.16(\mathrm{~s}, 3 \mathrm{H}), 3.13-3.03(\mathrm{~m}, 2 \mathrm{H}), 2.98(\mathrm{t}, J=7.1 \mathrm{~Hz}, 1 \mathrm{H}), 1.87$ $(\mathrm{m}, 2 \mathrm{H}), 1.80(\mathrm{~m}, 2 \mathrm{H}), 0.75(\mathrm{~d}, J=6.7 \mathrm{~Hz}, 3 \mathrm{H})$.

${ }^{13} \mathrm{C}$ NMR $\left(126 \mathrm{MHz}, \mathrm{CDCl}_{3}\right): \delta=177.7,169.5,145.1,136.5,135.4$, 133.7, 131.3, 130.2, 129.1, 127.6, 126.7, 126.3, 126.2, 126.0, 122.7, 109.0, 78.6, 48.3, 45.6, 44.1, 26.3, 25.9, 24.5, 13.8.

HRMS (ESI): $m / z[\mathrm{M}+\mathrm{H}]^{+}$calcd for $\mathrm{C}_{24} \mathrm{H}_{26} \mathrm{ClN}_{2} \mathrm{O}_{3}{ }^{+}$: 425.1626; found: 425.1632 .

Benzyl (E)-3-Hydroxy-3-(4-(2-(methylcarbamoyl)phenyl)but-3en-2-yl)azetidine-1-carboxylate $(4 \mathrm{j})$

The general procedure was followed using $N$-methylbenzamide (13.5 $\mathrm{mg}, 0.100 \mathrm{mmol}, 1.00$ equiv) and benzyl 3-oxoazetidine-1-carboxylate ( $61.5 \mathrm{mg}, 0.300 \mathrm{mmol}, 3.00$ equiv). Purification by silica gel chromatography (40\% EtOAc in $\mathrm{CH}_{2} \mathrm{Cl}_{2}$ ) provided $\mathbf{4 j}$ ( $33.9 \mathrm{mg}, 86 \%$ yield) as a colorless oil.

IR (neat): 3317, 2965, 2881, 1689, 1631, 1412, 1357, $1355 \mathrm{~cm}^{-1}$.

${ }^{1} \mathrm{H} \mathrm{NMR}\left(600 \mathrm{MHz}, \mathrm{CDCl}_{3}\right): \delta=7.41-7.38(\mathrm{~m}, 1 \mathrm{H}), 7.37-7.34(\mathrm{~m}, 1 \mathrm{H})$, 7.33-7.31 (m, $2 \mathrm{H}), 7.31-7.30(\mathrm{~m}, 1 \mathrm{H}), 7.29-7.25(\mathrm{~m}, 3 \mathrm{H}), 7.24-7.18$ $(\mathrm{m}, 1 \mathrm{H}), 6.73(\mathrm{~d}, J=15.9 \mathrm{~Hz}, 1 \mathrm{H}), 6.34-6.11(\mathrm{~m}, 1 \mathrm{H}), 5.97-5.93(\mathrm{~m}$, $1 \mathrm{H}), 4.98(\mathrm{~s}, 2 \mathrm{H}), 3.95(\mathrm{~m}, 2 \mathrm{H}), 3.81(\mathrm{~m}, 2 \mathrm{H}), 2.91(\mathrm{~d}, J=4.9 \mathrm{~Hz}, 3 \mathrm{H})$, $2.58(\mathrm{~m}, 1 \mathrm{H}), 1.11(\mathrm{~d}, J=6.8 \mathrm{~Hz}, 3 \mathrm{H})$.

${ }^{13} \mathrm{C}$ NMR $\left(151 \mathrm{MHz}, \mathrm{CDCl}_{3}\right): \delta=170.4,156.6,136.4,135.9,134.8$, $132.2,130.4,130.2,128.5,128.1,127.9,127.3,127.2,126.9,72.8,66.8$, $60.7,60.0,43.9,26.8,13.3$.

HRMS (ESI): $m / z[\mathrm{M}+\mathrm{H}]^{+}$calcd for $\mathrm{C}_{23} \mathrm{H}_{27} \mathrm{~N}_{2} \mathrm{O}_{4}^{+}$: 395.1965; found: 395.1971.

\section{Ethyl $\left(2 R^{*}, 3 S^{*}, E\right)-2-H y d r o x y-3-m e t h y l-5-(2-(m e t h y l c a r b a m o y l)-$} phenyl)-2-phenylpent-4-enoate (4k)

The general procedure was followed using $N$-methylbenzamide (13.5 $\mathrm{mg}, 0.100 \mathrm{mmol}, 1.00$ equiv) and ethyl 2-oxo-2-phenylacetate (53.4 $\mathrm{mg}, 0.300 \mathrm{mmol}, 3.00$ equiv). Purification by silica gel chromatography (40\% EtOAc in hexane) provided $4 \mathbf{k}$ ( $27.2 \mathrm{mg}$, 74\% yield; $92: 8 \mathrm{dr}$ ) as a white solid; $\mathrm{mp} 133-135{ }^{\circ} \mathrm{C}$.

IR (neat): 3529, 3337, 3060, 2939, 2879, 1727, 1630, $1538 \mathrm{~cm}^{-1}$.

${ }^{1} \mathrm{H}$ NMR $\left(500 \mathrm{MHz}, \mathrm{CDCl}_{3}\right): \delta=7.75(\mathrm{~d}, J=7.2 \mathrm{~Hz}$, minor, $0.26 \mathrm{H}), 7.69$ $(\mathrm{d}, J=7.3 \mathrm{~Hz}, 1.74 \mathrm{H}$, major), $7.49(\mathrm{~d}, J=7.9 \mathrm{~Hz}, 1 \mathrm{H}), 7.43(\mathrm{t}, J=7.8 \mathrm{~Hz}$, $1 \mathrm{H}), 7.38-7.35(\mathrm{~m}, 3 \mathrm{H}), 7.29(\mathrm{~m}, 1 \mathrm{H}), 7.26-7.23(\mathrm{~m}, 1 \mathrm{H}), 6.92(\mathrm{~d}, J=$ $15.9 \mathrm{~Hz}, 0.92 \mathrm{H}$, major), $6.52(\mathrm{~d}, J=15.9 \mathrm{~Hz}, 0.08 \mathrm{H}$, minor), 6.22 (dd, $J=15.9,8.8 \mathrm{~Hz}, 1 \mathrm{H}), 5.89$ (br s, $1 \mathrm{H}), 4.24-4.10(\mathrm{~m}, 2 \mathrm{H}), 3.83(\mathrm{~s}, 1 \mathrm{H})$, 3.35-3.29 (m, $1 \mathrm{H}), 3.01(\mathrm{~d}, J=4.7 \mathrm{~Hz}, 2.77 \mathrm{H}$, major), 2.91 (d, $J=4.8$ $\mathrm{Hz}, 0.23 \mathrm{H}$, minor), 1.32 (t, $J=7.1 \mathrm{~Hz}, 0.23 \mathrm{H}$, minor), 1.23 (d, $J=7.1$ $\mathrm{Hz}, 2.77 \mathrm{H}$, major), $1.20-1.19$ ( $\mathrm{m}, 0.23 \mathrm{H}$, minor), 0.91 (d, $J=6.8 \mathrm{~Hz}$, $2.77 \mathrm{H}$, major). 
${ }^{13} \mathrm{C}$ NMR $\left(126 \mathrm{MHz}, \mathrm{CDCl}_{3}\right): \delta=175.0,170.0,140.2,135.6,135.0$, 133.7, 130.2, 129.2, 128.2, 127.6, 127.5, 127.3, 126.7, 126.0, 80.6, 45.6, $26.7,14.12,14.06$; only peaks for the major isomer are listed.

HRMS (ESI): $m / z[\mathrm{M}+\mathrm{H}]^{+}$calcd for $\mathrm{C}_{22} \mathrm{H}_{26} \mathrm{NO}_{4}^{+}$: 368.1862; found: 368.1843.

\section{(E)-3-Hydroxy-1-methyl-3-(4-(4-methyl-2-(1H-pyrazol-1-yl)- phenyl)but-3-en-2-yl)indolin-2-one (4l)}

The general procedure was followed using $1-(m$-tolyl $)-1 H$-pyrazole (15.7 mg, $0.100 \mathrm{mmol}, 1.00$ equiv) and 1-methylindoline-2,3-dione ( $48.3 \mathrm{mg}, 0.300 \mathrm{mmol}, 3.00$ equiv). Purification by silica gel chromatography (50\% EtOAc in hexane) provided 41 (18.0 $\mathrm{mg}, 48 \%$ yield; $>20: 1 \mathrm{dr}$ ) as a light yellow solid; $\mathrm{mp} 155-157^{\circ} \mathrm{C}$.

IR (neat): 3341, 2962, 2324, 1699, 1612, 1460, $1373 \mathrm{~cm}^{-1}$.

${ }^{1} \mathrm{H} \mathrm{NMR}\left(400 \mathrm{MHz}, \mathrm{CDCl}_{3}\right): \delta=7.66(\mathrm{~s}, 1 \mathrm{H}), 7.56(\mathrm{~m}, 1 \mathrm{H}), 7.47(\mathrm{~d}, J=$ $7.8 \mathrm{~Hz}, 1 \mathrm{H}), 7.30(\mathrm{t}, J=7.8 \mathrm{~Hz}, 1 \mathrm{H}), 7.22-7.17(\mathrm{~m}, 3 \mathrm{H}), 7.02(\mathrm{t}, J=7.5$ $\mathrm{Hz}, 1 \mathrm{H}), 6.80(\mathrm{~d}, J=7.8 \mathrm{~Hz}, 1 \mathrm{H}), 6.40-6.37(\mathrm{~m}, 2 \mathrm{H}), 6.18(\mathrm{dd}, J=15.9$, $7.9 \mathrm{~Hz}, 1 \mathrm{H}), 3.17(\mathrm{~s}, 3 \mathrm{H}), 2.96-2.90(\mathrm{~m}, 1 \mathrm{H}), 2.38$ (s, $3 \mathrm{H}), 0.76$ (d, $J=$ $6.7 \mathrm{~Hz}, 3 \mathrm{H})$.

${ }^{13} \mathrm{C}$ NMR $\left(101 \mathrm{MHz}, \mathrm{CDCl}_{3}\right): \delta=177.3,143.8,140.8,138.4,138.0$, 131.1, 130.6, 129.8, 129.5, 129.3, 129.0, 128.5, 127.1, 126.1, 124.7, 122.8, 108.2, 106.5, 79.0, 44.4, 26.1, 21.0, 13.7 .

HRMS (ESI): $m / z[\mathrm{M}+\mathrm{H}]^{+}$calcd for $\mathrm{C}_{23} \mathrm{H}_{24} \mathrm{~N}_{3} \mathrm{O}_{2}{ }^{+}$: 374.1869; found: 374.1866 .

\section{Ethyl $\left(2 R^{*}, 3 S^{*}, E\right)$-2-Hydroxy-3-methyl-5-(4-methyl-2-(pyrimidin- 2-yl)phenyl)-2-phenylpent-4-enoate ( $4 \mathrm{~m})$}

The general procedure was followed using 2-( $m$-tolyl)pyrimidine (17.0 mg, $0.100 \mathrm{mmol}, 1.00$ equiv) and ethyl 2-oxo-2-phenylacetate (53.4 mg, $0.300 \mathrm{mmol}, 3.00$ equiv). Purification by silica gel chromatography (50\% EtOAc in hexane) provided $4 \mathrm{~m}(28.1 \mathrm{mg}$, 70\% yield; $>20: 1 \mathrm{dr}$ ) as a colorless oil.

IR (neat): 3995, 2974, 2928, 1725, 1568, 1555, $1420 \mathrm{~cm}^{-1}$.

${ }^{1} \mathrm{H} \mathrm{NMR}\left(500 \mathrm{MHz}, \mathrm{CDCl}_{3}\right): \delta=8.89(\mathrm{~d}, J=4.9 \mathrm{~Hz}, 2 \mathrm{H}), 7.70(\mathrm{~d}, J=7.7$ Hz, $2 \mathrm{H}), 7.65$ (s, $1 \mathrm{H}), 7.48$ (d, J = $7.9 \mathrm{~Hz}, 1 \mathrm{H}), 7.36(\mathrm{~m}, 2 \mathrm{H}), 7.25(\mathrm{~m}$, $3 \mathrm{H}), 7.03(\mathrm{~d}, J=15.9 \mathrm{~Hz}, 1 \mathrm{H}), 6.14(\mathrm{dd}, J=15.9,8.2 \mathrm{~Hz}, 1 \mathrm{H}), 4.11-$ $4.04(\mathrm{~m}, 2 \mathrm{H}), 3.30(\mathrm{~m}, 1 \mathrm{H}), 2.40(\mathrm{~s}, 3 \mathrm{H}), 1.10$ (t, J = 7.1 Hz, $3 \mathrm{H}), 0.88$ $(\mathrm{d}, J=6.8 \mathrm{~Hz}, 3 \mathrm{H})$.

${ }^{13} \mathrm{C}$ NMR $\left(126 \mathrm{MHz}, \mathrm{CDCl}_{3}\right): \delta=174.9,167.2,157.0,140.2,137.2$, $136.3,134.2,131.2,131.1,130.9,130.6,128.0,127.4,127.1,126.1$, 118.6, 80.7, 62.3, 45.2, 21.1, 14.0, 13.7.

HRMS (ESI): $m / z[M+\mathrm{H}]^{+}$calcd for $\mathrm{C}_{25} \mathrm{H}_{27} \mathrm{~N}_{2} \mathrm{O}_{3}{ }^{+}:$403.2016; found: 403.2022.

\section{Benzyl (E)-3-Hydroxy-3-(4-(4-methyl-2-(pyrimidin-2-yl)- phenyl)but-3-en-2-yl)azetidine-1-carboxylate (4n)}

The general procedure was followed using 2-( $m$-tolyl)pyrimidine (17.0 mg, $0.100 \mathrm{mmol}, 1.00$ equiv) and benzyl 3-oxoazetidine-1-carboxylate ( $61.5 \mathrm{mg}, 0.300 \mathrm{mmol}, 3.00$ equiv). Purification by silica gel chromatography (50\% EtOAc in hexane) provided 4 n $(25.7 \mathrm{mg}, 59 \%$ yield) as a colorless oil.

IR (neat): 3392, 2961, 2361, 1656, 1569, 1555, 1421, $1355 \mathrm{~cm}^{-1}$.

${ }^{1} \mathrm{H}$ NMR $\left(400 \mathrm{MHz}, \mathrm{CDCl}_{3}\right): \delta=8.79(\mathrm{~d}, J=4.9 \mathrm{~Hz}, 2 \mathrm{H}), 7.82(\mathrm{~d}, J=1.9$ $\mathrm{Hz}, 1 \mathrm{H}), 7.38-7.25(\mathrm{~m}, 7 \mathrm{H}), 7.21(\mathrm{~m}, 1 \mathrm{H}), 6.87(\mathrm{~d}, J=15.9 \mathrm{~Hz}, 1 \mathrm{H})$, $5.84(\mathrm{dd}, J=15.9,7.6 \mathrm{~Hz}, 1 \mathrm{H}), 5.09(\mathrm{~s}, 2 \mathrm{H}), 4.04(\mathrm{~m}, 2 \mathrm{H}), 3.88(\mathrm{~m}$, $2 \mathrm{H}), 2.65(\mathrm{~m}, 1 \mathrm{H}), 2.40(\mathrm{~s}, 3 \mathrm{H}), 1.16(\mathrm{~d}, J=6.9 \mathrm{~Hz}, 3 \mathrm{H})$.
${ }^{13} \mathrm{C}$ NMR $\left(126 \mathrm{MHz}, \mathrm{CDCl}_{3}\right): \delta=166.2,157.0,156.5,137.6,136.7$, $135.4,134.8,134.5,131.2,131.1,129.3,128.5,128.0,127.9,127.8$, 118.7, 73.0, 66.7, 60.4, 59.1, 43.5, 21.1, 13.2.

HRMS (ESI): $m / z[M+H]^{+}$calcd for $\mathrm{C}_{26} \mathrm{H}_{28} \mathrm{~N}_{3} \mathrm{O}_{3}{ }^{+}:$430.2125; found: 430.2131 .

(E)-3-Hydroxy-3-(4-(4-methyl-2-(pyrimidin-2-yl)phenyl)but-3en-2-yl)-5-nitroindolin-2-one (40)

The general procedure was followed using 2-( $m$-tolyl)pyrimidine (17.0 mg, $0.100 \mathrm{mmol}, 1.00$ equiv) and 5-nitroindoline-2,3-dione ( $57.6 \mathrm{mg}, 0.300 \mathrm{mmol}, 3.00$ equiv). Purification by silica gel chromatography (50\% EtOAc in $\mathrm{CH}_{2} \mathrm{Cl}_{2}$ ) provided 40 (34.2 $\mathrm{mg}, 82 \%$ yield; $85: 15 \mathrm{dr}$ ) as a yellow powder.

IR (neat): 3226, 2361, 1733, 1624, 1523, 1423, $1338 \mathrm{~cm}^{-1}$.

${ }^{1} \mathrm{H} \mathrm{NMR}\left(500 \mathrm{MHz}, \mathrm{CDCl}_{3}\right): \delta=8.87-8.85(\mathrm{~d}, J=4.9 \mathrm{~Hz}, 0.32 \mathrm{H}$, minor), $8.85-8.81$ (d, $J=4.9 \mathrm{~Hz}, 1.70 \mathrm{H}$, major), 8.51 (s, $0.82 \mathrm{H}$, major), 8.20 (s, $0.18 \mathrm{H}$, minor), $8.19(\mathrm{~m}, 1 \mathrm{H}), 8.00(\mathrm{~m}, 1 \mathrm{H}), 7.91(\mathrm{~s}, 0.86 \mathrm{H}$, major), $7.81(\mathrm{~s}, 0.18 \mathrm{H}$, minor), $7.42(\mathrm{~d}, J=7.9 \mathrm{~Hz}, 0.82 \mathrm{H}$, major), 7.37 (d, $J=$ $7.9 \mathrm{~Hz}, 0.18 \mathrm{H}$, minor), 7.32 (d, $J=7.9 \mathrm{~Hz}, 0.83 \mathrm{H}$, major), $7.29(\mathrm{~m}$, $0.18 \mathrm{H}$, minor), 7.24 (t, $J=4.9 \mathrm{~Hz}, 1 \mathrm{H}), 7.14(\mathrm{~d}, J=15.7 \mathrm{~Hz}, 0.88 \mathrm{H}$, major), 6.98 (d, $J=15.7 \mathrm{~Hz}, 0.18 \mathrm{H}$, minor), $6.92(\mathrm{~d}, J=8.6 \mathrm{~Hz}, 0.89 \mathrm{H}$, major), 6.87 (d, $J=8.6 \mathrm{~Hz}, 0.18 \mathrm{H}$, minor), 6.05 (dd, $J=15.7,9.2 \mathrm{~Hz}$, $0.15 \mathrm{H}$, minor), 5.93 (dd, $J=15.7,9.2 \mathrm{~Hz}, 0.85 \mathrm{H}$, major), 3.10-2.93 (m, $1 \mathrm{H}), 2.45$ (s, 2.67 H, major), $2.42(\mathrm{~s}, 0.57 \mathrm{H}$, minor), $1.25(\mathrm{~s}, 0.81 \mathrm{H}$, major), $1.04(\mathrm{~s}, 0.19 \mathrm{H}$, minor), $0.92(\mathrm{~d}, J=7.0 \mathrm{~Hz}, 3 \mathrm{H})$.

${ }^{13} \mathrm{C}$ NMR $\left(126 \mathrm{MHz}, \mathrm{CDCl}_{3}\right): \delta=179.0,165.9,157.1,146.5,143.6$, 138.1, 137.8, 135.3, 134.3, 131.40, 131.35, 131.0, 128.0, 127.4, 126.3, $120.7,118.7,109.9,79.6,45.0,21.2,14.0$; only peaks for the major isomer are listed.

HRMS (ESI): $m / z[\mathrm{M}+\mathrm{H}]^{+}$calcd for $\mathrm{C}_{23} \mathrm{H}_{21} \mathrm{~N}_{4} \mathrm{O}_{4}{ }^{+}$: 417.1557; found: 417.1563.

(E)-1-Benzyl-6-chloro-3-hydroxy-3-(4-(4-methyl-2-(pyrimidin-2yl)phenyl)but-3-en-2-yl)indolin-2-one (4p)

The general procedure was followed using 2-( $m$-tolyl $)$ pyrimidine (17.0 mg, $0.100 \mathrm{mmol}, 1.00$ equiv) and 1-benzyl-6-chloroindoline2,3-dione ( $81.5 \mathrm{mg}, 0.300 \mathrm{mmol}, 3.00$ equiv). Purification by silica gel chromatography (50\% EtOAc in hexane) provided $\mathbf{4 p}$ (32.2 $\mathrm{mg}, 65 \%$ yield; 88:12 $\mathrm{dr}$ ) as a yellow powder.

IR (neat): 3371, 3033, 2925, 1723, 1608, 1568, 1555, 1487, 1436, $1422,1374 \mathrm{~cm}^{-1}$.

${ }^{1} \mathrm{H}$ NMR (500 MHz, CDCl $): \delta=8.83(\mathrm{~d}, J=5.0 \mathrm{~Hz}, 2 \mathrm{H}), 7.93(\mathrm{~s}, 0.81 \mathrm{H}$, major), $7.92(\mathrm{~s}, 0.12 \mathrm{H}$, minor), $7.43(\mathrm{~d}, J=7.8 \mathrm{~Hz}, 1 \mathrm{H}), 7.37-7.27(\mathrm{~m}$, $6 \mathrm{H}), 7.24-7.15(\mathrm{~m}, 2 \mathrm{H}), 7.09(\mathrm{~d}, J=8.0 \mathrm{~Hz}, 1 \mathrm{H}), 7.04(\mathrm{~d}, J=7.9 \mathrm{~Hz}$, $0.18 \mathrm{H}$, minor), $6.90(\mathrm{~d}, J=7.9 \mathrm{~Hz}, 0.83 \mathrm{H}$, major), $6.71(\mathrm{~d}, J=1.8 \mathrm{~Hz}$, $0.88 \mathrm{H}$, major), 6.67 (d, $J=1.8 \mathrm{~Hz}, 0.12 \mathrm{H}$, minor), 6.04 (dd, $J=15.8,9.0$ $\mathrm{Hz}, 0.12 \mathrm{H}$, minor), 5.94 (dd, $J=15.8,9.0 \mathrm{~Hz}, 0.84 \mathrm{H}$, major), 5.05 (d, $J=$ $15.6 \mathrm{~Hz}, 0.86 \mathrm{H}$, major), 4.94 (d, $J=15.6 \mathrm{~Hz}, 0.15 \mathrm{H}$, minor), 4.74 (d, $J=$ $15.6 \mathrm{~Hz}, 0.85 \mathrm{H}$, major), 4.64 (d, $J=15.6 \mathrm{~Hz}, 0.15 \mathrm{H}$, minor), 3.10 (m, $0.88 \mathrm{H}$, major), 2.90 (m, $0.12 \mathrm{H}$, minor), 2.45 (s, $2.64 \mathrm{H}$, major), $2.42(\mathrm{~s}$, $0.36 \mathrm{H}$, minor), 0.97 (d, $J=6.8 \mathrm{~Hz}, 0.36 \mathrm{H}, \operatorname{minor}), 0.84(\mathrm{~d}, J=6.8 \mathrm{~Hz}$, $2.64 \mathrm{H}$, major).

${ }^{13} \mathrm{C}$ NMR $\left(126 \mathrm{MHz}, \mathrm{CDCl}_{3}\right): \delta=177.0,165.9,157.2,157.0,144.3$, 137.8, 136.3, 135.4, 135.1, 135.0, 134.8, 131.3, 131.2, 129.1, 128.92, $128.85,128.0,127.9,127.4,127.3,125.6,124.7,122.9,122.6,118.7$, 118.6, 109.81, 109.78, 79.2, 46.4, 44.7, 44.1, 43.9, 21.2, 21.1, 14.6, 14.3; peaks for both major and minor isomer are listed.

HRMS (ESI): $m / z[\mathrm{M}+\mathrm{H}]^{+}$calcd for $\mathrm{C}_{30} \mathrm{H}_{27} \mathrm{ClN}_{3} \mathrm{O}_{2}^{+}:$496.1786; found: 496.1792. 


\section{Funding Information}

This work was supported by the National Institutes of Health (R35GM122473)..

\section{Acknowledgment}

The helpful early input provided by Soham Maity is greatly appreciated.

\section{Supporting Information}

Supporting information for this article is available online at https://doi.org/10.1055/s-0039-1690741.

\section{References}

(1) Selected relevant reviews on $\mathrm{C}-\mathrm{H}$ functionalization: (a) Woźniak, Ł.; Cramer, N. Trends Chem. 2019, 1, 471. (b) Gandeepan, P.; Müller, T.; Zell, D.; Cera, G.; Warratz, S.; Ackermann, L. Chem. Rev. 2019, 119, 2192. (c) Kim, D.-S.; Park, W.-J.; Jun, C.-H. Chem. Rev. 2017, 117, 8977. (d) Hummel, J. R.; Boerth, J. A.; Ellman, J. A. Chem. Rev. 2017, 117, 9163. (e) Gensch, T.; Hopkinson, M. N.; Glorius, F.; Wencel-Delord, J. Chem. Soc. Rev. 2016, 45, 2900. (f) Yang, L.; Huang, H. Chem. Rev. 2015, 115, 3468. (g) Wencel-Delord, J.; Glorius, F. Nat. Chem. 2013, 5, 369. (h) Yamaguchi, J.; Yamaguchi, A. D.; Itami, K. Angew. Chem. Int. Ed. 2012, 51, 8960; Angew. Chem. 2012, 124, 9092.

(2) Selected reviews for three-component Catellani-type reactions: (a) Wang, J.; Dong, G. Chem. Rev. 2019, 119, 7478. (b) Della Ca', N.; Fontana, M.; Motti, E.; Catellani, M. Acc. Chem. Res. 2016, 49, 1389. (c) Ye, J.; Lautens, M. Nat. Chem. 2015, 7, 863. (d) Martins, A.; Mariampillai, B.; Lautens, M. Top. Curr. Chem. 2010, 292, 1. (e) Catellani, M. Top. Organomet. Chem. 2005, 14, 21. (f) Catellani, M.; Frignani, F.; Rangoni, A. Angew. Chem., Int. Ed. Engl. 1997, 36, 119; Angew. Chem. 1997, 109, 142.

(3) (a) Pinkert, T.; Wegner, T.; Mondal, S.; Glorius, F. Angew. Chem. Int. Ed. 2019, 58, 15041; Angew. Chem. 2019, 131, 15183. (b) Wang, X.-G.; Li, Y.; Liu, H.-C.; Zhang, B.-S.; Gou, X.-Y.; Wang, Q.; Ma, J.-W.; Liang, Y.-M. J. Am. Chem. Soc. 2019, 141, 13914. (c) Maity, S.; Potter, T. J.; Ellman, J. A. Nat. Catal. 2019, 2, 756. (d) Yang, J.; Ji, D.-W.; Hu, Y.-C.; Min, X.-T.; Zhou, X.; Chen, Q.-A. Chem. Sci. 2019, 10, 9560. (e) Dongbang, S.; Shen, Z.; Ellman, J. A. Angew. Chem. Int. Ed. 2019, 58, 12590; Angew. Chem. 2019, 131, 12720. (f) Li, R.; Ju, C.-W.; Zhao, D. Chem. Commun. 2019, 55, 695. (g) Tang, M.; Li, Y.; Han, S.; Liu, L.; Ackermann, L.; Li, J. Eur. J. Org. Chem. 2019, 660. (h) Boerth, J. A.; Maity, S.; Williams, S. K.; Mercado, B. Q.; Ellman, J. A. Nat. Catal. 2018, 1, 673. (i) Boerth, J. A.; Ellman, J. A. Angew. Chem. Int. Ed. 2017, 56, 9976; Angew. Chem. 2017, 129, 10108. (j) Zhang, S.-S.; Xia, J.; Wu, J.-Q.; Liu, X.-G.; Zhou, C.-J.; Lin, E.; Li, Q.; Huang, S.-L.; Wang, H. Org. Lett. 2017, 19, 5868. (k) Boerth, J. A.; Hummel, J. R.; Ellman, J. A. Angew. Chem. Int. Ed. 2016, 55, 12650; Angew. Chem. 2016, 128, 12840. (1) Boerth, J. A.; Ellman, J. A. Chem. Sci. 2016, 7, 1474.

(4) Intermolecular addition of unactivated $\mathrm{C}-\mathrm{H}$ bonds to ketones to give tertiary alcohols has only been reported for a couple of very highly activated ketones: (a) Jo, H.; Park, J.; Choi, M.; Sharma, S.; Jeon, M.; Mishra, N. K.; Jeong, T.; Han, S.; Kim, I. S. Adv. Synth. Catal. 2016, 358, 2714. (b) Zhang, X. S.; Zhu, Q. L.; Luo, F. X.; Chen, G. H.; Wang, X.; Shi, Z. J. Eur. J. Org. Chem. 2013, 6530.

(5) Tanaka, R.; Ikemoto, H.; Kanai, M.; Yoshino, T.; Matsunaga, S. Org. Lett. 2016, 18, 5732.

(6) CCDC 1956911 (4k) and CCDC 1957083 (4l) contain the supplementary crystallographic data for this paper. The data can be obtained free of charge from The Cambridge Crystallographic Data Centre via www.ccdc.cam.ac.uk/getstructures.

(7) Sheldrick, G. M. Acta Crystallogr., Sect. A 2008, 64, 112. 